\title{
NONNEGATIVITY OF THE CR PANEITZ OPERATOR FOR EMBEDDABLE CR MANIFOLDS
}

\author{
YUYA TAKEUCHI
}

\begin{abstract}
The nonnegativity of the CR Paneitz operator plays a crucial role in three-dimensional CR geometry. In this paper, we prove this nonnegativity for embeddable CR manifolds. This result gives an affirmative solution to the CR Yamabe problem for embeddable CR manifolds. We also show the existence of a contact form with zero CR $Q$-curvature and generalize the total $Q$-prime curvature to embeddable CR manifolds with no pseudo-Einstein contact forms. Furthermore, we discuss the logarithmic singularity of the Szegö kernel.
\end{abstract}

\section{INTRODUCTION}

For three-dimensional strictly pseudoconvex CR manifolds, the nonnegativity of the CR Paneitz operator has been of great importance; it is deeply connected to global embeddability [CCY12] and the CR positive mass theorem [CMY17]. It is known that there are closed non-embeddable CR manifolds of dimension three with not nonnegative CR Paneitz operator; see [CMY17, Section 4.1] for example. Hence it is natural to ask whether any closed embeddable CR three-manifold has nonnegative CR Paneitz operator. In this direction, Chanillo, Chiu, and Yang [CCY13] have proved that the nonnegativity condition is stable under sufficiently small deformations of strictly pseudoconvex real hypersurfaces in $\mathbb{C}^{2}$. Moreover, Case, Chanillo, and Yang [CCY16] have shown that the nonnegativity of the CR Paneitz operator is preserved under real-analytic embeddable deformations with uniform positivity of the Tanaka-Webster scalar curvature and the stability of the CR pluriharmonic functions. In this paper, we prove that the CR Paneitz operator is nonnegative for embeddable CR manifolds. Indeed:

Theorem 1.1. Let $\left(M, T^{1,0} M\right)$ be a closed embeddable strictly pseudoconvex $C R$ manifold of dimension three. Then the CR Paneitz operator is nonnegative, and its kernel consists of CR pluriharmonic functions.

Note that the kernel of the CR Paneitz operator has been studied by Case, Chanillo, and Yang [CCY15, CCY16] for deformations of embeddable CR threemanifolds.

Here we give an outline of the proof of Theorem 1.1 given in Section 4; our proof is inspired by that of the Siu-Sampson result for harmonic maps [Siu80, Sam86] in $\left[\mathrm{ABC}^{+} 96\right.$, Chapter 6.1]. Let $\left(M, T^{1,0} M\right)$ be as in Theorem 1.1. From [HL75,

2010 Mathematics Subject Classification. Primary 32V20; Secondary 32V15, 32V30, 53C55.

Key words and phrases. CR Paneitz operator, CR pluriharmonic function, CR $Q$-curvature, asymptotically complex hyperbolic manifold, Szegő kernel.

This work was supported by JSPS Research Fellowship for Young Scientists and JSPS KAKENHI Grant Number JP19J00063. 
Lem95], it follows that $M$ bounds a strictly pseudoconvex domain $\Omega$ in a twodimensional complex projective manifold $X$. By using a Kähler form on $X$ and a defining function of $\Omega$, we have an asymptotically complex hyperbolic Kähler form $\omega_{+}$on $\Omega$; see Section 4 for details. For $u \in C^{\infty}(M)$, take a harmonic extension $\widetilde{u}$ of $u$ to $\Omega$. Since $\omega_{+}$is Kähler, $\widetilde{u}$ satisfies $d d^{c} \widetilde{u} \wedge \omega_{+}=0$, where $d^{c}=(\sqrt{-1} / 2)(\bar{\partial}-\partial)$. Then we have $d d^{c} \widetilde{u} \wedge d d^{c} \widetilde{u} \leq 0$, and so

$$
\int_{M} d^{c} \widetilde{u} \wedge d d^{c} \widetilde{u}=\int_{\Omega} d d^{c} \widetilde{u} \wedge d d^{c} \widetilde{u} \leq 0
$$

On the other hand, integration by parts on $M$ yields that

$$
\int_{M} d^{c} \widetilde{u} \wedge d d^{c} \widetilde{u}=-\int_{M} u\left(P_{\theta} u\right) \theta \wedge d \theta
$$

where $\theta$ is a contact form on $M$ and $P_{\theta}$ is the CR Paneitz operator. Thus we obtain the nonnegativity of $P_{\theta}$. Moreover, if the equality holds, then $\widetilde{u}$ must be pluriharmonic, and $u$ is $\mathrm{CR}$ pluriharmonic.

Theorem 1.1 has various applications. Let $\left(M, T^{1,0} M\right)$ be a closed strictly pseudoconvex CR manifold of dimension three. The $C R$ Yamabe constant $\mathcal{Y}\left(M, T^{1,0} M\right)$ is defined by

$$
\mathcal{Y}\left(M, T^{1,0} M\right)=\inf _{\theta}\left\{\int_{M} \text { Scal } \cdot \theta \wedge d \theta \mid \int_{M} \theta \wedge d \theta=1\right\} .
$$

This value gives a CR invariant of $\left(M, T^{1,0} M\right)$. Theorem 1.1 and [CCY12, Theorem 1.4(b)] imply the following embeddability criterion.

Corollary 1.2. Let $\left(M, T^{1,0} M\right)$ be a closed strictly pseudoconvex CR manifold of dimension three with positive CR Yamabe constant. Then $\left(M, T^{1,0} M\right)$ is embeddable if and only if the CR Paneitz operator is nonnegative.

It is interesting to ask whether we can remove the positivity of the CR Yamabe constant from the assumption of Corollary 1.2.

It is known [JL87] that the CR Yamabe constant satisfies

$$
\mathcal{Y}\left(M, T^{1,0} M\right) \leq \mathcal{Y}\left(S^{3}, T^{1,0} S^{3}\right),
$$

where $\left(S^{3}, T^{1,0} S^{3}\right)$ is the standard CR sphere in $\mathbb{C}^{2}$; note that $\mathcal{Y}\left(S^{3}, T^{1,0} S^{3}\right)>$ 0. Combining Theorem 1.1 with a consequence of the $\mathrm{CR}$ positive mass theorem [CMY17, Theorem 1.2], we have the following

Corollary 1.3. Let $\left(M, T^{1,0} M\right)$ be a closed embeddable three-dimensional strictly pseudoconvex $C R$ manifold. Then $\mathcal{Y}\left(M, T^{1,0} M\right)=\mathcal{Y}\left(S^{3}, T^{1,0} S^{3}\right)$ if and only if $\left(M, T^{1,0} M\right)$ is $C R$ equivalent to $\left(S^{3}, T^{1,0} S^{3}\right)$.

The CR Yamabe problem is to find a contact form $\theta$ satisfying

$$
\text { Scal }=\mathcal{Y}\left(M, T^{1,0} M\right), \quad \int_{M} \theta \wedge d \theta=1 ;
$$

such a contact form is called a CR Yamabe contact form. Recently, Cheng, Malchiodi, and Yang [CMY19] have found that there exist no CR Yamabe contact forms on the Rossi sphere, which is an example of non-embeddable CR manifolds. Nonetheless, the CR Yamabe problem is solvable affirmatively for embeddable three-dimensional CR manifolds.

Theorem 1.4. There exists a CR Yamabe contact form on any closed embeddable strictly pseudoconvex CR manifold of dimension three. 
We will also apply Theorem 1.1 to the zero CR $Q$-curvature problem. In a study of the Szegö kernel, Hirachi [Hir93] has introduced a pseudo-Hermitian invariant

$$
Q_{\theta}=\frac{1}{6}\left(\Delta_{b} \mathrm{Scal}-2 \operatorname{Im} A_{11}{ }^{11}\right),
$$

which is now called the $C R Q$-curvature. Note that this name stems from the fact that this invariant coincides with a $\mathrm{CR}$ analog of the $Q$-curvature; see [FH03]. Since $Q_{\theta}$ is expressed as a divergence, its integral is identically zero. Moreover, the $\mathrm{CR} Q$-curvature itself is identically zero for pseudo-Einstein contact forms; that is, contact forms satisfying the equality

$$
\mathrm{Scal}_{1}-\sqrt{-1} A_{11}{ }^{1}=0
$$

The existence of such a contact form is equivalent to that of a Fefferman defining function if $M$ bounds a strictly pseudoconvex domain [Hir93,HPT08]; in particular, it always exists if the $\mathrm{CR}$ manifold is a real hypersurface in $\mathbb{C}^{2}$. For these reasons, it is natural to ask whether any CR manifold admits a contact form with zero $\mathrm{CR} Q$-curvature. Some authors have tackled this problem via the $\mathrm{CR} Q$-curvature flow [CCC07, SC11, CKS19]. In this paper, however, we will take a more functionalanalytic approach.

Theorem 1.5. Let $\left(M, T^{1,0} M\right)$ be a closed embeddable strictly pseudoconvex $C R$ manifold of dimension three. There exists a contact form $\theta$ on $M$ with zero $C R$ $Q$-curvature. Moreover, if $\hat{\theta}$ is also such a contact form, then $\hat{\theta}=e^{\Upsilon} \theta$ for a $C R$ pluriharmonic function $\Upsilon$. In particular, in the case where $M$ admits a pseudoEinstein contact form, $\theta$ is pseudo-Einstein if and only if $Q_{\theta}=0$.

The existence of such a contact form gives a generalization of the total $Q$-prime curvature to embeddable $\mathrm{CR}$ manifolds with no pseudo-Einstein contact forms. Let $\left(M, T^{1,0} M\right)$ be a closed strictly pseudoconvex $\mathrm{CR}$ manifold of dimension three and $\theta$ a contact form on $M$. The $Q$-prime curvature $Q_{\theta}^{\prime}$ is defined by

$$
Q_{\theta}^{\prime}=\frac{1}{2} \mathrm{Scal}^{2}-2\left|A_{11}\right|^{2}+\Delta_{b} \text { Scal }
$$

Case and Yang [CY13] have proved that the integral of the $Q$-prime curvature

$$
\bar{Q}^{\prime}\left(M, T^{1,0} M\right)=\int_{M} Q_{\theta}^{\prime} \theta \wedge d \theta
$$

is independent of the choice of a contact form $\theta$ with zero CR $Q$-curvature, and defines a $\mathrm{CR}$ invariant of $\left(M, T^{1,0} M\right)$, called the total $Q$-prime curvature. Hence, if $\left(M, T^{1,0} M\right)$ is embeddable, then the total $Q$-prime curvature is always welldefined and gives a CR invariant. The total $Q$-prime curvature and the CR Yamabe constant satisfy the following inequality, which is a CR analog of [Gur99, Equation (1.4)].

Theorem 1.6. Let $\left(M, T^{1,0} M\right)$ be a closed embeddable three-dimensional strictly pseudoconvex CR manifold. Then

$$
\bar{Q}^{\prime}\left(M, T^{1,0} M\right) \leq \frac{1}{2} \mathcal{Y}\left(M, T^{1,0} M\right)^{2}
$$

with equality if and only if $M$ admits a pseudo-Einstein contact form with vanishing Tanaka-Webster torsion. In particular if $c_{1}\left(T^{1,0} M\right) \neq 0$ in $H^{2}(M, \mathbb{R})$, then the strict inequality holds. 
Note that there exists a two-dimensional strictly pseudoconvex domain whose boundary $M$ satisfies $c_{1}\left(T^{1,0} M\right) \neq 0$ in $H^{2}(M, \mathbb{R})$ [EO08, Theorem 6.2]. In particular, the strict inequality in (1.1) holds for this $M$.

We also mention the case where the equality in (1.1) holds. A CR manifold having a pseudo-Einstein contact form with vanishing Tanaka-Webster torsion is known as a Sasakian $\eta$-Einstein manifold. Such a manifold plays a crucial role in CR geometry; see [Wan15, CG17, Tak18] for example.

Theorem 1.6 gives a generalization of [CY13, Theorem 1.1] for embeddable CR manifolds.

Corollary 1.7. Let $\left(M, T^{1,0} M\right)$ be a closed embeddable strictly pseudoconvex $C R$ manifold of dimension three with nonnegative CR Yamabe constant. Then

$$
\bar{Q}^{\prime}\left(M, T^{1,0} M\right) \leq \bar{Q}^{\prime}\left(S^{3}, T^{1,0} S^{3}\right)
$$

with equality if and only if $\left(M, T^{1,0} M\right)$ is CR equivalent to $\left(S^{3}, T^{1,0} S^{3}\right)$.

We will also apply our results to the logarithmic singularity of the Szegö kernel. Let $\Omega$ be a strictly pseudoconvex domain in a two-dimensional complex manifold and $\theta$ a contact form on $\partial \Omega$. Define the Hardy space $\mathcal{H}_{\theta}(\Omega)$ as the set consisting of the boundary values of holomorphic functions on $\Omega$ that are $L^{2}$ with respect to the volume form $\theta \wedge d \theta$ on $\partial \Omega$. The Szegö kernel $S_{\theta}(z, \bar{w})$ is the reproducing kernel of $\mathcal{H}_{\theta}(\Omega)$. Fix a defining function $\rho$ of $\Omega$. The boundary behavior of $S_{\theta}$ on the diagonal is given by

$$
S_{\theta}(z, \bar{z})=\phi_{\theta} \rho^{-2}+\psi_{\theta} \log (-\rho),
$$

where $\phi_{\theta}$ and $\psi_{\theta}$ are smooth functions on $\bar{\Omega}$. Moreover, the Tayler coefficients of $\psi_{\theta}$ at a given boundary point are uniquely determined by the behavior of $\theta$ near the point [BdMS76]. Hirachi [Hir93, Main Theorem] has proved that

$$
\left.\psi_{\theta}\right|_{\partial \Omega}=\frac{1}{4 \pi^{2}} Q_{\theta} .
$$

Hence $\psi_{\theta}=O(\rho)$ if and only if $\theta$ is of zero CR $Q$-curvature. It is natural to ask when $\psi_{\theta}$ vanishes to higher-order on the boundary.

Theorem 1.8. Let $\Omega$ be a two-dimensional strictly pseudoconvex domain whose boundary admits a pseudo-Einstein contact form. For a contact form $\theta$ on $\partial \Omega$, the function $\psi_{\theta}$ is of $O\left(\rho^{2}\right)$ (resp. $O\left(\rho^{3}\right)$ ) if and only if $\theta$ is pseudo-Einstein and $\partial \Omega$ is obstruction flat (resp. spherical).

Note that the above theorem has been obtained by Hirachi in the case when $\Omega$ is a domain in $\mathbb{C}^{2}$ and $\partial \Omega$ has transverse symmetry [Hir93]. It is interesting to ask what happens when $\partial \Omega$ has no pseudo-Einstein contact forms. We also mention a recent progress by Curry and Ebenfelt [CE18, CE19] on the question: If $\psi_{\theta}=O\left(\rho^{2}\right)$, does $\psi_{\theta}=O\left(\rho^{3}\right)$ hold?

This paper is organized as follows. In Section 2, we recall some basic definitions and facts on CR manifolds. In Section 3, we discuss a CR analog of $d^{c}$, which is closely related to $\mathrm{CR}$ pluriharmonic functions and plays an important role in the proofs of our results in this paper. Section 4 is devoted to proofs of Theorems 1.1 and 1.4. In Section 5, we tackle the existence problem of a contact form with zero CR $Q$-curvature. Section 6 deals with the logarithmic singularity of the Szegö kernel. 


\section{Preliminaries}

Let $M$ be a smooth three-dimensional manifold without boundary. A $C R$ structure is a complex one-dimensional subbundle $T^{1,0} M$ of the complexified tangent bundle $T M \otimes \mathbb{C}$ such that

$$
T^{1,0} M \cap T^{0,1} M=0,
$$

where $T^{0,1} M$ is the complex conjugate of $T^{1,0} M$ in $T M \otimes \mathbb{C}$. An important example of a three-dimensional CR manifold is a real hypersurface $M$ in a two-dimensional complex manifold $X$; this $M$ has the canonical CR structure

$$
T^{1,0} M=\left.T^{1,0} X\right|_{M} \cap(T M \otimes \mathbb{C}) .
$$

Introduce an operator $\bar{\partial}_{b}: C^{\infty}(M) \rightarrow \Gamma\left(\left(T^{0,1} M\right)^{*}\right)$ by

$$
\bar{\partial}_{b} f=\left.(d f)\right|_{T^{0,1} M} .
$$

A smooth function $f$ is called a $C R$ holomorphic function if $\bar{\partial}_{b} f=0$. A $C R$ pluriharmonic function is a real-valued smooth function that is locally the real part of a CR holomorphic function. We denote by $\mathcal{P}$ the space of $\mathrm{CR}$ pluriharmonic functions.

A CR structure $T^{1,0} M$ is said to be strictly pseudoconvex if there exists a nowhere-vanishing real one-form $\theta$ on $M$ such that $\theta$ annihilates $T^{1,0} M$ and

$$
-\sqrt{-1} d \theta(Z, \bar{Z})>0, \quad 0 \neq Z \in T^{1,0} M ;
$$

we call such a one-form a contact form. Denote by $T$ the Reeb vector field with respect to $\theta$; that is, the unique vector field satisfying

$$
\theta(T)=1, \quad \iota_{T} d \theta=0 .
$$

Let $Z_{1}$ be a local frame of $T^{1,0} M$, and set $Z_{\overline{1}}=\overline{Z_{1}}$. Then $\left(T, Z_{1}, Z_{\overline{1}}\right)$ gives a local frame of $T M \otimes \mathbb{C}$, called an admissible frame. Its dual frame $\left(\theta, \theta^{1}, \theta^{\overline{1}}\right)$ is called an admissible coframe. The two-form $d \theta$ is written as

$$
d \theta=\sqrt{-1} l_{1 \overline{1}} \theta^{1} \wedge \theta^{\overline{1}}
$$

where $l_{1 \overline{1}}$ is a positive function. We use $l_{1 \overline{1}}$ and its multiplicative inverse $l^{1 \overline{1}}$ to raise and lower indices.

A contact form $\theta$ induces a canonical connection $\nabla$, called the Tanaka-Webster connection with respect to $\theta$. It is defined by

$$
\nabla T=0, \quad \nabla Z_{1}=\omega_{1}^{1} Z_{1}, \quad \nabla Z_{\overline{1}}=\omega_{\overline{1}}{ }^{\overline{1}} Z_{\overline{1}} \quad\left(\omega_{\overline{1}}{ }^{\overline{1}}=\overline{\omega_{1}^{1}}\right)
$$

with the following structure equations:

$$
\begin{gathered}
d \theta^{1}=\theta^{1} \wedge \omega_{1}{ }^{1}+A^{1} \overline{\overline{1}}_{\overline{1}} \theta \wedge \theta^{\overline{1}}, \\
d l_{1 \overline{1}}=\omega_{1}{ }^{1} l_{1 \overline{1}}+l_{1 \overline{1}} \omega_{\overline{1}}{ }^{\overline{1}} .
\end{gathered}
$$

The tensor $A_{11}=\overline{A_{\overline{11}}}$ is called the Tanaka-Webster torsion. We denote the components of a successive covariant derivative of a tensor by subscripts preceded by a comma, for example, $K_{1 \overline{1}, 1}$; we omit the comma if the derivatives are applied to a function. We use the index 0 for the component $T$ or $\theta$ in our index notation. The commutators of the second derivatives for $u \in C^{\infty}(M)$ are given by

$$
u_{1 \overline{1}}-u_{\overline{1} 1}=\sqrt{-1} l_{1 \overline{1}} u_{0}, \quad u_{01}-u_{10}=A_{11} u^{1}
$$


see [Lee88, (2.14)]. Define the sub-Laplacian $\Delta_{b}$ by

$$
\Delta_{b} u=-u_{\overline{1}}^{\overline{1}}-u_{1}^{1}
$$

for $u \in C^{\infty}(M)$. From (2.1), it follows that

$$
\Delta_{b} u=-2 u_{\overline{1}}^{{ }^{1}}-\sqrt{-1} u_{0}=-2 u_{1}{ }^{1}+\sqrt{-1} u_{0} .
$$

The curvature form $\Omega_{1}{ }^{1}=d \omega_{1}{ }^{1}$ of the Tanaka-Webster connection satisfies

$$
\Omega_{1}{ }^{1}=\mathrm{Scal} \cdot l_{1 \overline{1}} \theta^{1} \wedge \theta^{\overline{1}}-A_{11}{ }^{1} \theta \wedge \theta^{1}+A_{\overline{11}}{ }^{\overline{1}} \theta \wedge \theta^{\overline{1}},
$$

where Scal is the Tanaka-Webster scalar curvature.

Let $\hat{\theta}=e^{\Upsilon} \theta$ be another contact form, and denote by $\widehat{T}$ the corresponding Reeb vector field. The admissible coframe corresponding to $\left(\widehat{T}, Z_{1}, Z_{\overline{1}}\right)$ is given by

$$
\left(\hat{\theta}, \hat{\theta}^{1}=\theta^{1}+\sqrt{-1} \Upsilon^{1} \theta, \hat{\theta}^{\overline{1}}=\theta^{\overline{1}}-\sqrt{-1} \Upsilon^{\overline{1}} \theta\right) .
$$

Under this conformal change, the sub-Laplacian $\widehat{\Delta}_{b}$ with respect to $\hat{\theta}$ satisfies

$$
e^{\Upsilon} \widehat{\Delta}_{b} u=\Delta_{b} u-\Upsilon^{1} u_{1}-\Upsilon^{\overline{1}} u_{\overline{1}} ;
$$

see [Sta89, Lemma 1.8] for example.

An important example of strictly pseudoconvex CR manifolds is the boundary of a strictly pseudoconvex domain. Let $\Omega$ be a relatively compact domain in a two-dimensional complex manifold $X$ with smooth boundary $M=\partial \Omega$. Then there exists a smooth function $\rho$ on $X$ such that

$$
\Omega=\rho^{-1}((-\infty, 0)), \quad M=\rho^{-1}(0), \quad d \rho \neq 0 \text { on } M ;
$$

such a $\rho$ is called a defining function of $\Omega$. A domain $\Omega$ is said to be strictly pseudoconvex if we can take a defining function $\rho$ of $\Omega$ such that $d d^{c} \rho$ defines a Kähler form near $M$. The boundary of a strictly pseudoconvex domain is a closed strictly pseudoconvex real hypersurface; the one-form $\left.d^{c} \rho\right|_{M}$ is a contact form on $M$.

\section{CR PLURIHARMONIC FUnCTIONS AND PSEUdO-EINSTEIN CONDITION}

Let $\left(M, T^{1,0} M\right)$ be a strictly pseudoconvex CR manifold of dimension three and $\theta$ a contact form on $M$. We first discuss a CR analog of $d^{c}$. Such an operator was first introduced by Rumin [Rum94] in his study of the Rumin complex. Here, however, we define it as an operator taking values in $\Omega^{1}(M)$, which implicitly appears in the proof of [Hir14, Lemma 3.2].

Lemma 3.1. The differential operator

$$
d_{\mathrm{CR}}^{c}: C^{\infty}(M) \rightarrow \Omega^{1}(M) ; \quad u \mapsto \frac{\sqrt{-1}}{2}\left(u_{\overline{1}} \theta^{\overline{1}}-u_{1} \theta^{1}\right)+\frac{1}{2} \Delta_{b} u \cdot \theta
$$

is independent of the choice of $\theta$.

Proof. Consider the conformal change $\hat{\theta}=e^{\Upsilon} \theta$. The independence of $d_{\mathrm{CR}}^{c}$ in $\theta$ follows from the transformation laws of an admissible coframe (2.5) and the subLaplacian (2.6).

As in complex geometry, CR pluriharmonic functions are smooth functions annihilated by $d d_{\mathrm{CR}}^{c}$. 
Lemma 3.2. For $u \in C^{\infty}(M)$,

$$
d d_{\mathrm{CR}}^{c} u=P_{1} u \cdot \theta \wedge \theta^{1}+P_{\overline{1}} u \cdot \theta \wedge \theta^{\overline{1}},
$$

where

$$
P_{1} u=u_{\overline{1}^{\overline{1}}{ }_{1}}+\sqrt{-1} A_{11} u^{1}, \quad P_{\overline{1}} u=u_{1}^{1}{ }_{\overline{1}}-\sqrt{-1} A_{\overline{11}} u^{\overline{1}} .
$$

In particular, $u$ is a CR pluriharmonic function if and only if $d d_{\mathrm{CR}}^{c} u=0$.

Proof. We first show the equality (3.2). From (2.2) and (3.1), it follows that

$$
\begin{aligned}
d d_{\mathrm{CR}}^{c} u=[ & \left.-\frac{1}{2}\left(\Delta_{b} u\right)_{1}-\frac{\sqrt{-1}}{2} u_{10}+\frac{\sqrt{-1}}{2} A_{11} u^{1}\right] \theta \wedge \theta^{1} \\
& +\left[-\frac{1}{2}\left(\Delta_{b} u\right)_{\overline{1}}+\frac{\sqrt{-1}}{2} u_{\overline{10}}-\frac{\sqrt{-1}}{2} A_{\overline{11}} u^{\overline{1}}\right] \theta \wedge \theta^{\overline{1}} .
\end{aligned}
$$

Hence it suffices to show

$$
P_{1} u=-\frac{1}{2}\left(\Delta_{b} u\right)_{1}-\frac{\sqrt{-1}}{2} u_{10}+\frac{\sqrt{-1}}{2} A_{11} u^{1} ;
$$

the other part is the complex conjugate of this equality. By using (2.1) and (2.3), we have

$$
\begin{aligned}
-\frac{1}{2}\left(\Delta_{b} u\right)_{1}-\frac{\sqrt{-1}}{2} u_{10}+\frac{\sqrt{-1}}{2} A_{11} u^{1} & =u_{\overline{1}}{ }^{\overline{1}}{ }_{1}+\frac{\sqrt{-1}}{2}\left(u_{01}-u_{10}\right)+\frac{\sqrt{-1}}{2} A_{11} u^{1} \\
& =u_{\overline{1}}{ }^{\overline{1}}{ }_{1}+\sqrt{-1} A_{11} u^{1} \\
& =P_{1} u .
\end{aligned}
$$

The latter statement is a consequence of [Lee88, Proposition 3.4].

Each $u \in \mathcal{P}$ defines a cohomology class $\left[d_{\mathrm{CR}}^{c} u\right]$ in $H^{1}(M, \mathbb{R})$. The following lemma is obtained from [Lee88, Lemma 3.1].

Lemma 3.3. For $u \in \mathcal{P}$, the cohomology class $\left[d_{\mathrm{CR}}^{c} u\right]$ is equal to zero if and only if $u$ is the real part of a CR holomorphic function globally.

Assume that $M$ is realized as the boundary of a two-dimensional strictly pseudoconvex domain $\Omega$. Take a defining function $\rho$ of $\Omega$ with $\theta=\left.d^{c} \rho\right|_{M}$.

Lemma 3.4. For each $u \in C^{\infty}(M)$, there exists a smooth extension $\widetilde{u}$ of $u$ to $\bar{\Omega}$ such that $\left.d d^{c} \widetilde{u}\right|_{M}$ has vanishing $(1,1)$-part. Moreover, such a $\widetilde{u}$ is unique modulo $O\left(\rho^{2}\right)$, and $\left.d^{c} \widetilde{u}\right|_{M}$ coincides with $d_{\mathrm{CR}}^{c} u$.

Proof. Take a smooth function $u^{\prime}$ on $\bar{\Omega}$ with $\left.u^{\prime}\right|_{M}=u$. Then

$$
\left.d^{c} u^{\prime}\right|_{M}=\frac{\sqrt{-1}}{2}\left(u_{\overline{1}} \theta^{\overline{1}}-u_{1} \theta^{1}\right)+\lambda \theta
$$

for some $\lambda \in C^{\infty}(M)$. Hence the $(1,1)$-part of $\left.d d^{c} u^{\prime}\right|_{M}$ is given by

$$
\frac{\sqrt{-1}}{2}\left(u_{\overline{1} 1}+u_{1 \overline{1}}+2 \lambda l_{1 \overline{1}}\right) \theta^{1} \wedge \theta^{\overline{1}} \text {. }
$$

On the other hand, the $(1,1)$-part of $\left.d d^{c}(\rho v)\right|_{M}$ for $v \in C^{\infty}(\bar{\Omega})$ coincides with

$$
\left.\sqrt{-1} v\right|_{M} l_{1 \overline{1}} \theta^{1} \wedge \theta^{\overline{1}} \text {. }
$$

If we choose $v$ so that $\left.v\right|_{M}=2^{-1} \Delta_{b} u-\lambda$, the $(1,1)$-part of $\left.d d^{c}\left(u^{\prime}+\rho v\right)\right|_{M}$ vanishes, which gives the existence of $\widetilde{u}$. From the construction, it follows that $\left.d^{c} \widetilde{u}\right|_{M}=d_{\mathrm{CR}}^{c} u$. The uniqueness of $\widetilde{u}$ modulo $O\left(\rho^{2}\right)$ is a consequence of the above computation of the (1,1)-part of $\left.d d^{c}(\rho v)\right|_{M}$. 
Set

$$
W_{1}=\mathrm{Scal}_{1}-\sqrt{-1} A_{11},{ }^{1}, \quad W_{\overline{1}}=\overline{W_{1}}=\mathrm{Scal}_{\overline{1}}+\sqrt{-1} A_{\overline{11}}{ }^{\overline{1}},
$$

and define a two-form $W(\theta)$ on $M$ by

$$
W(\theta)=W_{1} \theta \wedge \theta^{1}+W_{\overline{1}} \theta \wedge \theta^{\overline{1}} .
$$

A contact form $\theta$ is said to be pseudo-Einstein if $W(\theta)=0$.

Lemma 3.5. The two-form $W(\theta) / 2 \pi$ is closed and gives a representative of the first Chern class $c_{1}\left(T^{1,0} M\right)$. In particular, $c_{1}\left(T^{1,0} M\right)=0$ in $H^{2}(M, \mathbb{R})$ if $\left(M, T^{1,0} M\right)$ admits a pseudo-Einstein contact form.

Proof. It follows from (2.4) that

$$
W(\theta)=\sqrt{-1} \Omega_{1}{ }^{1}-d(\text { Scal } \cdot \theta)=\sqrt{-1} d \omega_{1}{ }^{1}-d(\text { Scal } \cdot \theta) .
$$

Hence $W(\theta) / 2 \pi$ is closed and represents the cohomology class $c_{1}\left(T^{1,0} M\right)$.

The two-form $W(\theta)$ depends on the choice of $\theta$, but $d d_{\mathrm{CR}}^{c}$ appears in its transformation law under conformal change.

Lemma 3.6. For another contact form $\hat{\theta}=e^{\Upsilon} \theta$,

$$
W(\hat{\theta})=W(\theta)-3 d d_{\mathrm{CR}}^{c} \Upsilon .
$$

In particular, in the case that $\theta$ is pseudo-Einstein, so is $\hat{\theta}$ if and only if $\Upsilon$ is $C R$ pluriharmonic.

Proof. Under the conformal change $\hat{\theta}=e^{\Upsilon} \theta$,

$$
e^{\Upsilon} \widehat{W}_{1}=W_{1}-3 P_{1} \Upsilon
$$

see [Hir93, Lemma 5.4] for example. Hence

$$
W(\hat{\theta})=\widehat{W}_{1} \hat{\theta} \wedge \hat{\theta}^{1}+\widehat{W}_{\overline{1}} \hat{\theta} \wedge \hat{\theta}^{\overline{1}}=W(\theta)-3 d d_{\mathrm{CR}}^{c} \Upsilon,
$$

which establishes the formula.

\section{CR Paneitz operator in Dimension three}

Let $\left(M, T^{1,0} M\right)$ be a closed three-dimensional strictly pseudoconvex CR manifold and $\theta$ a contact form on $M$. The CR Paneitz operator $P_{\theta}$ is defined by

$$
P_{\theta} u=\left(P_{1} u\right)^{1}=\left(P_{\overline{1}} u\right)^{\overline{1}} \text {. }
$$

The CR Paneitz operator is formally self-adjoint and annihilates CR pluriharmonic functions [GL88, Section 2]. We first show an integral formula for $P_{\theta}$ by using $d_{\mathrm{CR}}^{c}$.

Lemma 4.1. For $u \in C^{\infty}(M)$,

$$
\int_{M} u\left(P_{\theta} u\right) \theta \wedge d \theta=-\int_{M} d_{\mathrm{CR}}^{c} u \wedge d d_{\mathrm{CR}}^{c} u .
$$

Proof. From the definition of $d_{\mathrm{CR}}^{c}(3.1)$ and Lemma 3.2, it follows that

$$
\begin{aligned}
\int_{M} d_{\mathrm{CR}}^{c} u \wedge d d_{\mathrm{CR}}^{c} u & =\frac{\sqrt{-1}}{2} \int_{M}\left[u_{\overline{1}}\left(P_{1} u\right) \theta^{\overline{1}} \wedge \theta \wedge \theta^{1}-u_{1}\left(P_{\overline{1}} u\right) \theta^{1} \wedge \theta \wedge \theta^{\overline{1}}\right] \\
& =\frac{1}{2} \int_{M}\left[u^{1}\left(P_{1} u\right)+u^{\overline{1}}\left(P_{\overline{1}} u\right)\right] \theta \wedge d \theta \\
& =-\int_{M} u\left(P_{\theta} u\right) \theta \wedge d \theta,
\end{aligned}
$$


which completes the proof.

Assume that $\left(M, T^{1,0} M\right)$ is embeddable; this means $\left(M, T^{1,0} M\right)$ can be embedded in $\mathbb{C}^{N}$ for large $N$. Without loss of generality, we may assume that $M$ is connected. Harvey and Lawson [HL75, Theorem 10.4] have shown that $M$ bounds a two-dimensional strictly pseudoconvex Stein space. Lempert [Lem95, Theorem 8.1] has proved that, in this case, $M$ can be realized as the boundary of a strictly pseudoconvex domain $\Omega$ in a two-dimensional complex projective manifold $X$. Take a Kähler form $\omega$ on $X$ and a defining function $\rho$ of $\Omega$. For sufficiently large $N>0$, the real $(1,1)$-form

$$
\omega_{+}=N \omega-2 d d^{c} \log (-\rho)
$$

defines an (even) asymptotically complex hyperbolic metric on $\Omega$; see [EMM91, GSB08, Mat16] for details. Denote by $\Delta_{+}$the (nonnegative) Riemannian Laplacian for $\omega_{+}$.

Lemma 4.2. Let $F$ be a smooth function on $\bar{\Omega}$ with $\left.F\right|_{M}=u$. The condition $\Delta_{+} F=O\left(\rho^{2}\right)$ is equivalent to that $\left.d d^{c} F\right|_{M}$ has vanishing $(1,1)$-part. In particular, $\left.d^{c} F\right|_{M}=d_{\mathrm{CR}}^{c} u$ if $\Delta_{+} F=O\left(\rho^{2}\right)$.

Proof. We first note that

$$
-4 d d^{c} F \wedge \omega_{+}=\left(\Delta_{+} F\right) \omega_{+}^{2} .
$$

It follows from the definition of $\omega_{+}$that

$$
\omega_{+}^{2}=-8 \rho^{-3} d \rho \wedge d^{c} \rho \wedge d d^{c} \rho+O\left(\rho^{-2}\right) .
$$

Take a local frame $\widetilde{Z}_{1}$ of $T^{1,0} X \cap \operatorname{Ker} \partial \rho$, and set $\widetilde{Z}_{\overline{1}}=\widetilde{Z}_{1}$. Since $M$ is strictly pseudoconvex, $d d^{c} \rho\left(\widetilde{Z}_{1}, \widetilde{Z}_{\overline{1}}\right)$ is non-zero near $M$, and

$$
d d^{c} F \wedge \omega_{+}=2 \rho^{-2} \frac{d d^{c} F\left(\widetilde{Z}_{1}, \widetilde{Z}_{\overline{1}}\right)}{d d^{c} \rho\left(\widetilde{Z}_{1}, \widetilde{Z}_{\overline{1}}\right)} d \rho \wedge d^{c} \rho \wedge d d^{c} \rho+O\left(\rho^{-1}\right) .
$$

This proves the former statement. The latter one is a consequence of Lemma 3.4.

We need a solution to the Dirichlet problem for $\Delta_{+}$. Near $M$, the Laplacian $\Delta_{+}$ satisfies

$$
\Delta_{+}=-\left(\rho \frac{\partial}{\partial \rho}\right)^{2}+2 \rho \frac{\partial}{\partial \rho}+(\text { a diff. op. increasing the order in } \rho) ;
$$

c.f. [GL88, Proposition 2.1].

Proposition 4.3. For each $u \in C^{\infty}(M)$, there exist smooth functions $F$ and $G$ on $\bar{\Omega}$ such that $\left.F\right|_{M}=u$ and $\widetilde{u}=F+G \rho^{2} \log (-\rho)$ satisfies $\Delta_{+} \widetilde{u}=0$.

Proof. For a smooth function $f$ on $\bar{\Omega},(4.1)$ implies that

$$
\begin{aligned}
\Delta_{+}\left(f \rho^{k}\right) & =k(2-k) f \rho^{k}+O\left(\rho^{k+1}\right), \\
\Delta_{+}\left(f \rho^{k} \log (-\rho)\right) & =k(2-k) f \rho^{k} \log (-\rho)+2(1-k) f \rho^{k}+O\left(\rho^{k+1} \log (-\rho)\right) .
\end{aligned}
$$

By using an inductive argument and Borel's lemma, we obtain smooth functions $F_{0}$ and $G$ on $\bar{\Omega}$ such that $\left.F_{0}\right|_{M}=u$ and

$$
H=\Delta_{+}\left(F_{0}+G \rho^{2} \log (-\rho)\right) \in \rho^{\infty} C^{\infty}(\bar{\Omega}),
$$


where $\rho^{\infty} C^{\infty}(\bar{\Omega})$ is the space of smooth functions on $\bar{\Omega}$ that vanish to infinite order on $M$. Epstein, Melrose, and Mendoza [EMM91] have proved that $\Delta_{+}$has a bounded inverse $R$, and it maps $\rho^{\infty} C^{\infty}(\bar{\Omega})$ to $\rho^{2} C^{\infty}(\bar{\Omega})$; see also [GSB08, Section 5.1]. If we set $F=F_{0}-R(H)$, then we have $\left.F\right|_{M}=u$ and $\Delta_{+}\left(F+G \rho^{2} \log (-\rho)\right)=$ 0 .

Note that $\Delta_{+} F=O\left(\rho^{2}\right)$, and so $\left.d^{c} F\right|_{M}=d_{\mathrm{CR}}^{c} u$ by Lemma 4.2. Now we give a proof of the nonnegativity of the CR Paneitz operator.

Proof of Theorem 1.1. Fix a smooth function $u$ on $M$, and let $\widetilde{u}=F+G \rho^{2} \log (-\rho)$ be as in Proposition 4.3. Since $d d^{c} \widetilde{u} \wedge \omega_{+}=0$, we have

$$
d d^{c} \widetilde{u} \wedge d d^{c} \widetilde{u} \leq 0
$$

with equality if and only if $d d^{c} \widetilde{u}=0$. The one-form $d^{c}\left(G \rho^{2} \log (-\rho)\right)$ can be extended continuously to $\bar{\Omega}$ and vanishes along $M$. On the other hand,

$$
d d^{c}\left(G \rho^{2} \log (-\rho)\right) \equiv \rho^{2} \log (-\rho) d d^{c} G+(2 \rho \log (-\rho)+\rho) d\left(G d^{c} \rho\right) \quad(\bmod d \rho)
$$

The right hand side is continuous up to $M$ and equal to zero on $M$. By using Stokes' theorem, we have

$$
\begin{aligned}
\int_{M} d_{\mathrm{CR}}^{c} u \wedge d d_{\mathrm{CR}}^{c} u & =\int_{M} d^{c} F \wedge d d^{c} F \\
& =\lim _{\epsilon \rightarrow+0} \int_{\rho=-\epsilon} d^{c} \widetilde{u} \wedge d d^{c} \widetilde{u} \\
& =\lim _{\epsilon \rightarrow+0} \int_{\rho<-\epsilon} d d^{c} \widetilde{u} \wedge d d^{c} \widetilde{u} \\
& \leq 0 .
\end{aligned}
$$

Hence Lemma 4.1 yields the nonnegativity of the CR Paneitz operator. If the equality holds, then $\widetilde{u}$ must be pluriharmonic on $\Omega$, and so $d d^{c} \widetilde{u} \wedge d \rho=0$ on $\Omega$. From (4.2), it follows that

$$
d d^{c}\left(G \rho^{2} \log (-\rho)\right) \wedge d \rho=\left[\rho^{2} \log (-\rho) d d^{c} G+(2 \rho \log (-\rho)+\rho) d\left(G d^{c} \rho\right)\right] \wedge d \rho
$$

is continuous up to $M$ and vanishes along $M$. Therefore we have $d d^{c} F \wedge d \rho=0$ on $M$, which implies $\left.d d^{c} F\right|_{M}=d d_{\mathrm{CR}}^{c} u=0$. In particular, $u$ is annihilated by $P_{\theta}$ if and only if $u$ is CR pluriharmonic.

From the above proof, we obtain the following

Corollary 4.4. Any $C R$ pluriharmonic function $u$ on $M$ admits a smooth pluriharmonic extension $\widetilde{u}$ to $\bar{\Omega}$, and $\left.d^{c} \widetilde{u}\right|_{M}=d_{\mathrm{CR}}^{c} u$ holds.

Proof. Let $u$ be a CR pluriharmonic function on $M$, and $\widetilde{u}=F+G \rho^{2} \log (-\rho)$ be as in Proposition 4.3. From the proof of Theorem 1.1, it follows that $\widetilde{u}$ is pluriharmonic on $\Omega$; in other words, $d d^{c} \widetilde{u}=0$. On the other hand, $d d^{c} \widetilde{u}$ is equal to $2 G \log (-\rho) d \rho \wedge d^{c} \rho$ modulo a two-form continuous up to the boundary. Thus we have $\left.G\right|_{M}=0$. The construction of $G$ implies that $G \in \rho^{\infty} C^{\infty}(\bar{\Omega})$; in particular, $\widetilde{u}$ is smooth up to the boundary. The equality $\left.d^{c} \widetilde{u}\right|_{M}=d_{\mathrm{CR}}^{c} u$ follows from $d d^{c} \widetilde{u}=0$ and Lemma 3.4.

We apply Corollary 1.3, which follows from Theorem 1.1 and [CMY17, Theorem 1.2], to give an affirmative solution to the CR Yamabe problem for embeddable CR manifolds. 
Proof of Theorem 1.4. If $\mathcal{Y}\left(M, T^{1,0} M\right)<\mathcal{Y}\left(S^{3}, T^{1,0} S^{3}\right)$, then there exists a CR Yamabe contact form on $M$ [JL87, Theorem 3.4]. On the other hand, if $\mathcal{Y}\left(M, T^{1,0} M\right)=$ $\mathcal{Y}\left(S^{3}, T^{1,0} S^{3}\right)$, then $\left(M, T^{1,0} M\right)$ is CR equivalent to $\left(S^{3}, T^{1,0} S^{3}\right)$ by Corollary 1.3 , and a solution of the CR Yamabe problem exists [JL87, Theorem 7.2].

\section{CR $Q$-Curvature in Dimension three}

In this section, we discuss the zero CR $Q$-curvature problem in dimension three and some applications. Let $\left(M, T^{1,0} M\right)$ be a closed three-dimensional strictly pseudoconvex CR manifold and $\theta$ a contact form on $M$. Hirachi [Hir93] has introduced the $C R Q$-curvature $Q_{\theta}$ by

$$
Q_{\theta}=\frac{1}{6}\left(\Delta_{b} \text { Scal }-2 \operatorname{Im} A_{11,}{ }^{11}\right) .
$$

He has derived also the divergence formula

$$
Q_{\theta}=-\frac{1}{3} W_{1,}^{1}=-\frac{1}{3} W_{\overline{1}}^{\overline{1}}
$$

and its transformation law under conformal change $\hat{\theta}=e^{\Upsilon} \theta$ :

$$
e^{2 \Upsilon} Q_{\hat{\theta}}=Q_{\theta}+P_{\theta} \Upsilon
$$

see [Hir93, Lemma 5.4]. In particular, the CR $Q$-curvature vanishes for pseudoEinstein contact forms.

We first discuss orthogonality relations between $Q_{\theta}$ and $\mathcal{P}$. The integral of the product of a $\mathrm{CR}$ pluriharmonic function and the $\mathrm{CR} Q$-curvature has a cohomological expression.

Proposition 5.1. For $u \in \mathcal{P}$,

$$
\left\langle\left[d_{\mathrm{CR}}^{c} u\right] \cup c_{1}\left(T^{1,0} M\right),[M]\right\rangle=\frac{3}{2 \pi} \int_{M} u Q_{\theta} \theta \wedge d \theta .
$$

Proof. The definitions of $d_{\mathrm{CR}}^{c}(3.1)$ and $W(\theta)$ (3.3) yield that

$$
\begin{aligned}
\int_{M} d_{\mathrm{CR}}^{c} u \wedge W(\theta) & =\frac{\sqrt{-1}}{2} \int_{M}\left[u_{\overline{1}} W_{1} \theta^{\overline{1}} \wedge \theta \wedge \theta^{1}-u_{1} W_{\overline{1}} \theta^{1} \wedge \theta \wedge \theta^{\overline{1}}\right] \\
& =\frac{1}{2} \int_{M}\left[u^{1} W_{1}+u^{\overline{1}} W_{\overline{1}}\right] \theta \wedge d \theta \\
& =3 \int_{M} u Q_{\theta} \theta \wedge d \theta .
\end{aligned}
$$

Since $W(\theta) / 2 \pi$ is a representative of $c_{1}\left(T^{1,0} M\right)$, we have the desired conclusion.

Lemma 3.3 gives the following

Corollary 5.2. If $u$ is the real part of a CR holomorphic function globally, then $Q_{\theta}$ is orthogonal to $u$.

Similarly, the vanishing of the first Chern class implies the orthogonality of the CR $Q$-curvature to $\mathcal{P}$.

Corollary 5.3. If $c_{1}\left(T^{1,0} M\right)=0$ in $H^{2}(M, \mathbb{R})$, then $Q_{\theta}$ is orthogonal to $\mathcal{P}$.

Now we give a complete solution to the zero CR $Q$-curvature problem for embeddable CR manifolds. To this end, we recall some functional-analytic properties of the CR Paneitz operator obtained by Hsiao. 
Theorem 5.4 ([Hsi15, Theorem 1.2]). Let $\left(M, T^{1,0} M\right)$ be a closed embeddable strictly pseudoconvex $C R$ manifold of dimension three and $\theta$ a contact form on $M$. The maximal closed extension of $P_{\theta}$ is self-adjoint with closed range. Moreover, if $u \in \operatorname{Dom} P_{\theta} \cap\left(\operatorname{Ker} P_{\theta}\right)^{\perp}$ satisfies $P_{\theta} u \in C^{\infty}(M)$, then $u$ must be smooth.

Denote by $\Pi_{\theta}$ the orthogonal projection to Ker $P_{\theta}$. We write $G_{\theta}$ for the partial inverse of $P_{\theta}$; that is, $G_{\theta}$ is a bounded linear operator from $L^{2}(M)$ to $\operatorname{Dom} P_{\theta} \cap$ $\left(\text { Ker } P_{\theta}\right)^{\perp}$ satisfying

$$
\begin{gathered}
u=\Pi_{\theta} u+P_{\theta} G_{\theta} u \quad u \in L^{2}(M), \\
u=\Pi_{\theta} u+G_{\theta} P_{\theta} u \quad u \in \operatorname{Dom} P_{\theta} .
\end{gathered}
$$

From the latter statement of Theorem 5.4, it follows that $G_{\theta}$ maps $\operatorname{Ran} P_{\theta} \cap C^{\infty}(M)$ to itself, and $\Pi_{\theta}$ defines a linear operator $C^{\infty}(M) \rightarrow \operatorname{Ker} P_{\theta} \cap C^{\infty}(M)$; in particular, $\mathcal{P}=\operatorname{Ker} P_{\theta} \cap C^{\infty}(M)$ is dense in $\operatorname{Ker} P_{\theta}$.

Proof of Theorem 1.5. Fix a contact form $\theta_{0}$ on $M$. If $Q_{\theta_{0}}$ is orthogonal to $\mathcal{P}$, then

$$
\theta=\exp \left(-G_{\theta_{0}} Q_{\theta_{0}}\right) \cdot \theta_{0}
$$

is a contact form with zero CR $Q$-curvature. Hence it suffices to show

$$
\left\langle\left[d_{\mathrm{CR}}^{c} u\right] \cup c_{1}\left(T^{1,0} M\right),[M]\right\rangle=0
$$

for any $u \in \mathcal{P}$. Take a smooth pluriharmonic extension $\widetilde{u}$ of $u$ to $\bar{\Omega}$ by Corollary 4.4. Fix a Hermitian metric on $T^{1,0} \bar{\Omega}$, and denote by $\Psi$ the corresponding first Chern form. By applying Stokes' theorem, we have

$$
0=\int_{\Omega} d d^{c} \widetilde{u} \wedge \Psi=\left.\int_{M} d_{\mathrm{CR}}^{c} u \wedge \Psi\right|_{M}=\left\langle\left[d_{\mathrm{CR}}^{c} u\right] \cup c_{1}\left(T^{1,0} M\right),[M]\right\rangle ;
$$

here we use the fact that $\left.\Psi\right|_{M}$ is a representative of $c_{1}\left(T^{1,0} M\right)=c_{1}\left(\left.T^{1,0} \bar{\Omega}\right|_{M}\right)$. If $\hat{\theta}=e^{\Upsilon} \theta$ is also a contact form with zero CR $Q$-curvature, then $\Upsilon$ is annihilated by $P_{\theta}$, and so it is CR pluriharmonic by Theorem 1.1.

Case and Yang [CY13] have defined the $Q$-prime curvature $Q_{\theta}^{\prime}$ by

$$
Q_{\theta}^{\prime}=\frac{1}{2} \mathrm{Scal}^{2}-2\left|A_{11}\right|^{2}+\Delta_{b} \mathrm{Scal}
$$

For any contact forms $\theta$ and $\hat{\theta}=e^{\Upsilon} \theta$, the integral of the $Q$-prime curvature satisfies

$$
\int_{M} Q_{\hat{\theta}}^{\prime} \hat{\theta} \wedge d \hat{\theta}=\int_{M} Q_{\theta}^{\prime} \theta \wedge d \theta+6 \int_{M}\left[\Upsilon\left(P_{\theta} \Upsilon\right)+2 Q_{\theta} \Upsilon\right] \theta \wedge d \theta
$$

see [CY13, Proposition 6.1]. In particular, a discussion in [CY13] yields that the integral of the $Q$-prime curvature

$$
\bar{Q}^{\prime}\left(M, T^{1,0} M\right)=\int_{M} Q_{\theta}^{\prime} \theta \wedge d \theta
$$

is independent of the choice of a contact form $\theta$ with zero CR $Q$-curvature, and defines a CR invariant of $\left(M, T^{1,0} M\right)$, which we call the total $Q$-prime curvature. By Theorem 1.5, the total $Q$-prime curvature is always well-defined for closed embeddable CR three-manifolds. Now we give a proof of Theorem 1.6, which relates the total $Q$-prime curvature with the CR Yamabe constant. 
Proof of Theorem 1.6. Fix a contact form $\theta$ with zero CR $Q$-curvature. Theorem 1.4 implies that there exists $\Upsilon \in C^{\infty}(M)$ such that $\hat{\theta}=e^{\Upsilon} \theta$ satisfies

$$
\widehat{\mathrm{Scal}}=\mathcal{Y}\left(M, T^{1,0} M\right), \quad \int_{M} \hat{\theta} \wedge d \hat{\theta}=1 .
$$

Then

$$
\begin{aligned}
\int_{M} Q_{\hat{\theta}}^{\prime} \hat{\theta} \wedge d \hat{\theta} & =\frac{1}{2} \int_{M}(\widehat{\text { Scal }})^{2} \hat{\theta} \wedge d \hat{\theta}-2 \int_{M}\left|\widehat{A}_{11}\right|^{2} \hat{\theta} \wedge d \hat{\theta} \\
& \leq \frac{1}{2} \mathcal{Y}\left(M, T^{1,0} M\right)^{2}
\end{aligned}
$$

On the other hand, it follows from (5.1) and the nonnegativity of $P_{\theta}$ that

$$
\begin{aligned}
\int_{M} Q_{\hat{\theta}}^{\prime} \hat{\theta} \wedge d \hat{\theta} & =\bar{Q}^{\prime}\left(M, T^{1,0} M\right)+6 \int_{M} \Upsilon\left(P_{\theta} \Upsilon\right) \theta \wedge d \theta \\
& \geq \bar{Q}^{\prime}\left(M, T^{1,0} M\right) .
\end{aligned}
$$

Therefore we get the desired inequality. Moreover, if the equality holds, then $\hat{\theta}$ has vanishing Tanaka-Webster torsion, and it is a pseudo-Einstein contact form since

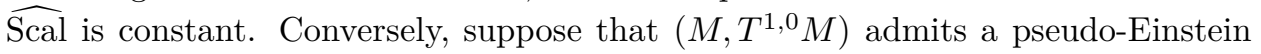
contact form $\theta$ with vanishing Tanaka-Webster torsion; in this case, the TanakaWebster scalar curvature is constant. Without loss of generality, we may assume that $\int_{M} \theta \wedge d \theta=1$. From Theorem 1.4 and [Wan15, Theorem 4], it follows that $\theta$ is a CR Yamabe contact form, and so the equality holds.

Theorem 1.6 gives a generalization of [CY13, Theorem 1.1] to embeddable CR manifolds.

Proof of Corollary 1.7. Since $0 \leq \mathcal{Y}\left(M, T^{1,0} M\right) \leq \mathcal{Y}\left(S^{3}, T^{1,0} S^{3}\right)$, we have

$$
\begin{aligned}
\mathcal{Y}\left(M, T^{1,0} M\right)^{2} & \leq \mathcal{Y}\left(S^{3}, T^{1,0} S^{3}\right)^{2} \\
& =2 \bar{Q}^{\prime}\left(S^{3}, T^{1,0} S^{3}\right) ;
\end{aligned}
$$

here the last equality follows from Theorem 1.6 and the fact that the standard contact form on $S^{3}$ is a pseudo-Einstein contact form with vanishing Tanaka-Webster torsion. Moreover, if the equality holds, then $\mathcal{Y}\left(M, T^{1,0} M\right)=\mathcal{Y}\left(S^{3}, T^{1,0} S^{3}\right)$, and $\left(M, T^{1,0} M\right)$ is CR equivalent to $\left(S^{3}, T^{1,0} S^{3}\right)$ by Corollary 1.3 .

\section{Logarithmic Singularity OF THE Szegő KeRnel}

Let $\Omega$ be a strictly pseudoconvex domain in a two-dimensional complex manifold $X$. We recall some facts on Fefferman defining functions; see [HPT08, Section 2D] for example. For a local coordinate $z=\left(z^{1}, z^{2}\right)$ of $X$, set

$$
\mathcal{J}_{z}[\varphi]=-\operatorname{det}\left(\begin{array}{cc}
\varphi & \partial \varphi / \partial \bar{z}^{b} \\
\partial \varphi / \partial z^{a} & \partial^{2} \varphi / \partial z^{a} \partial \bar{z}^{b}
\end{array}\right)
$$

If $w=F(z)$ is another local coordinate, then

$$
\mathcal{J}_{w}[\varphi]=\left|\operatorname{det} F^{\prime}\right|^{2} \mathcal{J}_{z}[\varphi],
$$

where $F^{\prime}$ is the holomorphic Jacobi matrix of $F$. A Fefferman defining function is a defining function $\rho$ of $\Omega$ such that

$$
d d^{c} \log \mathcal{J}_{z}[\rho]=d d^{c} O\left(\rho^{3}\right)
$$


for any local coordinate $z$. The condition (6.1) is equivalent to that, for any $p \in \partial \Omega$, there exists a local coordinate $z$ near $p$ such that

$$
\mathcal{J}_{z}[\rho]=1+O\left(\rho^{3}\right) .
$$

It is known that $\theta$ is a pseudo-Einstein contact form on $\partial \Omega$ if and only if $\theta=\left.d^{c} \rho\right|_{\partial \Omega}$ for a Fefferman defining function $\rho$ of $\Omega$; see [Hir93, Lemma 7.2] and [HPT08, Proposition 2.10] for example. We say $\partial \Omega$ to be obstruction flat if we can take a defining function $\rho$ of $\Omega$ such that $d d^{c} \log \mathcal{J}_{z}[\rho]$ vanishes to infinite order near a given point on $\partial \Omega$.

Now we give a necessary and sufficient condition for the vanishing of $\psi_{\theta}$ in (1.2) to higher-order on the boundary.

Proof of Theorem 1.8. From (1.3), it follows that $\left.\psi_{\theta}\right|_{\partial \Omega}=0$ is equivalent to $Q_{\theta}=$ 0 . Since $\partial \Omega$ has a pseudo-Einstein contact form, $\left.\psi_{\theta}\right|_{\partial \Omega}=0$ if and only if $\theta$ is pseudo-Einstein by Theorem 1.5. In what follows, we suppose that $\theta$ is pseudoEinstein. Take a Fefferman defining function $\rho$ with $\theta=\left.d^{c} \rho\right|_{\partial \Omega}$. For a point $p \in \partial \Omega$, take a local coordinate $z$ near $p$ such that $\mathcal{J}_{z}[\rho]=1+O\left(\rho^{3}\right)$. Hirachi, Komatsu, and Nakazawa [HKN93] have computed $\psi_{\theta}$ up to second order in this setting. From [Gra87, Proposition 1.8] and [HKN93, Proposition 1 and Theorem 1], it follows that $\psi_{\theta}=O\left(\rho^{2}\right)$ if and only if $\partial \Omega$ is obstruction flat. On the other hand, we derive from [HKN93, Remark 2] that $\psi_{\theta}=O\left(\rho^{3}\right)$ if and only if $\partial \Omega$ is spherical.

\section{ACKNOWLEDGEMENTS}

The author is grateful to Kengo Hirachi for valuable suggestions on the logarithmic singularity of the Szegő kernel. He would like to express his gratitude to Jeffrey Case, Sagun Chanillo, and Paul Yang for helpful comments. He would like to thank the referees for their comments also, which are helpful for the improvement of the manuscript.

\section{REFERENCES}

$\left[\mathrm{ABC}^{+96}\right]$ J. Amorós, M. Burger, K. Corlette, D. Kotschick, and D. Toledo, Fundamental groups of compact Kähler manifolds, Mathematical Surveys and Monographs, vol. 44, American Mathematical Society, Providence, RI, 1996.

[BdMS76] L. Boutet de Monvel and J. Sjöstrand, Sur la singularité des noyaux de Bergman et de Szegő, Journées: Équations aux Dérivées Partielles de Rennes (1975), 1976, pp. 123164. Astérisque, No. 34-35.

[CCC07] S.-C. Chang, J.-H. Cheng, and H.-L. Chiu, A fourth order curvature flow on a CR 3-manifold, Indiana Univ. Math. J. 56 (2007), no. 4, 1793-1826.

[CCY12] S. Chanillo, H.-L. Chiu, and P. Yang, Embeddability for 3-dimensional CauchyRiemann manifolds and CR Yamabe invariants, Duke Math. J. 161 (2012), no. 15, 2909-2921.

[CCY13] S. Chanillo, H.-L. Chiu, and P. Yang, Embedded three-dimensional CR manifolds and the non-negativity of Paneitz operators, Geometric analysis, mathematical relativity, and nonlinear partial differential equations, 2013, pp. 65-82.

[CCY15] J. S. Case, S. Chanillo, and P. Yang, A remark on the kernel of the CR Paneitz operator, Nonlinear Anal. 126 (2015), 153-158.

[CCY16] J. S. Case, S. Chanillo, and P. Yang, The CR Paneitz operator and the stability of CR pluriharmonic functions, Adv. Math. 287 (2016), 109-122.

[CE18] S. N. Curry and P. Ebenfelt, Bounded strictly pseudoconvex domains in $\mathbb{C}^{2}$ with obstruction flat boundary, 2018. arXiv: 1803.09053. 
[CE19] S. N. Curry and P. Ebenfelt, Bounded strictly pseudoconvex domains in $\mathbb{C}^{2}$ with obstruction flat boundary II, Adv. Math. 352 (2019), 611-631.

[CG17] J. S. Case and A. R. Gover, The $P^{\prime}$-operator, the $Q^{\prime}$-curvature, and the CR tractor calculus, 2017. arXiv:1709.08057, to appear in Ann. Sc. Norm. Super. Pisa Cl. Sci.

[CKS19] S.-C. Chang, T.-J. Kuo, and T. Saotome, Global existence and convergence for the CR Q-curvature flow in a closed strictly pseudoconvex CR 3-manifold, 2019. arXiv: 1905.01783.

[CMY17] J.-H. Cheng, A. Malchiodi, and P. Yang, A positive mass theorem in three dimensional Cauchy-Riemann geometry, Adv. Math. 308 (2017), 276-347.

[CMY19] J.-H. Cheng, A. Malchiodi, and P. Yang, On the Sobolev quotient of three-dimensional CR manifolds, 2019. arXiv:1904.04665.

[CY13] J. S. Case and P. Yang, A Paneitz-type operator for CR pluriharmonic functions, Bull. Inst. Math. Acad. Sin. (N.S.) 8 (2013), no. 3, 285-322.

[EMM91] C. L. Epstein, R. B. Melrose, and G. A. Mendoza, Resolvent of the Laplacian on strictly pseudoconvex domains, Acta Math. 167 (1991), no. 1-2, 1-106.

[EO08] J. B. Etnyre and B. Ozbagci, Invariants of contact structures from open books, Trans. Amer. Math. Soc. 360 (2008), no. 6, 3133-3151.

[FH03] C. Fefferman and K. Hirachi, Ambient metric construction of Q-curvature in conformal and CR geometries, Math. Res. Lett. 10 (2003), no. 5-6, 819-831.

[GL88] C. R. Graham and J. M. Lee, Smooth solutions of degenerate Laplacians on strictly pseudoconvex domains, Duke Math. J. 57 (1988), no. 3, 697-720.

[Gra87] C. R. Graham, Scalar boundary invariants and the Bergman kernel, Complex analysis, II (College Park, Md., 1985-86), 1987, pp. 108-135.

[GSB08] C. Guillarmou and A. Sá Barreto, Scattering and inverse scattering on ACH manifolds, J. Reine Angew. Math. 622 (2008), 1-55.

[Gur99] M. J. Gursky, The principal eigenvalue of a conformally invariant differential operator, with an application to semilinear elliptic PDE, Comm. Math. Phys. 207 (1999), no. $1,131-143$.

[Hir14] K. Hirachi, Q-prime curvature on CR manifolds, Differential Geom. Appl. 33 (2014), no. suppl., 213-245.

[Hir93] K. Hirachi, Scalar pseudo-Hermitian invariants and the Szegö kernel on threedimensional CR manifolds, Complex geometry (Osaka, 1990), 1993, pp. 67-76.

[HKN93] K. Hirachi, G. Komatsu, and N. Nakazawa, Two methods of determining local invariants in the Szegö kernel, Complex geometry (Osaka, 1990), 1993, pp. 77-96.

[HL75] F. R. Harvey and H. B. Lawson Jr., On boundaries of complex analytic varieties. I, Ann. of Math. (2) 102 (1975), no. 2, 223-290.

[HPT08] P. D. Hislop, P. A. Perry, and S.-H. Tang, CR-invariants and the scattering operator for complex manifolds with boundary, Anal. PDE 1 (2008), no. 2, 197-227.

[Hsi15] C.-Y. Hsiao, On CR Paneitz operators and CR pluriharmonic functions, Math. Ann. 362 (2015), no. 3-4, 903-929.

[JL87] D. Jerison and J. M. Lee, The Yamabe problem on CR manifolds, J. Differential Geom. 25 (1987), no. 2, 167-197.

[Lee88] J. M. Lee, Pseudo-Einstein structures on CR manifolds, Amer. J. Math. 110 (1988), no. $1,157-178$.

[Lem95] L. Lempert, Algebraic approximations in analytic geometry, Invent. Math. 121 (1995), no. 2, 335-353.

[Mat16] Y. Matsumoto, GJMS operators, Q-curvature, and obstruction tensor of partially integrable CR manifolds, Differential Geom. Appl. 45 (2016), 78-114.

[Rum94] M. Rumin, Formes différentielles sur les variétés de contact, J. Differential Geom. 39 (1994), no. 2, 281-330.

[Sam86] J. H. Sampson, Applications of harmonic maps to Kähler geometry, Complex differential geometry and nonlinear differential equations (Brunswick, Maine, 1984), 1986, pp. $125-134$.

[SC11] T. Saotome and S.-C. Chang, The Q-curvature flow in a closed CR 3-manifold, Proceedings of the 15th International Workshop on Differential Geometry and the 4th KNUGRG-OCAMI Differential Geometry Workshop [Volume 15], 2011, pp. 57-69. 
[Siu80] Y. T. Siu, The complex-analyticity of harmonic maps and the strong rigidity of compact Kähler manifolds, Ann. of Math. (2) 112 (1980), no. 1, 73-111.

[Sta89] N. K. Stanton, Spectral invariants of CR manifolds, Michigan Math. J. 36 (1989), no. $2,267-288$.

[Tak18] Y. Takeuchi, Ambient constructions for Sasakian $\eta$-Einstein manifolds, Adv. Math. $\mathbf{3 2 8}$ (2018), 82-111.

[Wan15] X. Wang, On a remarkable formula of Jerison and Lee in CR geometry, Math. Res. Lett. 22 (2015), no. 1, 279-299.

Department of Mathematics, Graduate School of Science, Osaka University, 1-1 MACHIKANEYAMA-CHO, TOYONAKA, OSAKA 560-0043, JAPAN

Email address: yu-takeuchi@cr.math.sci.osaka-u.ac.jp, yuya.takeuchi.math@gmail.com 